\title{
HYPOELLIPTIC DIFFERENTIAL OPERATORS WITH GENERALIZED CONSTANT COEFFICIENTS
}

\author{
by M. NEDELJKOV and S. PILIPOVIĆ \\ (Received 7th December 1995)
}

\begin{abstract}
The space $\mathcal{G}$ of Colombeau generalized functions is used as a frame for the study of hypoellipticity of a family of differential operators whose coefficients depend on a small parameter $\varepsilon$.

There are given necessary and sufficient conditions for the hypoellipticity of a family of differential operators with constant coefficients which depend on $\varepsilon$ and behave like powers of $\varepsilon$ as $\varepsilon \rightarrow 0$. The solutions of such family of equations should also satisfy the power order estimate with respect to $\varepsilon$.
\end{abstract}

1991 Mathematics subject classification: $35 \mathrm{Hxx}, 46 \mathrm{Fxx}$.

\section{Introduction}

Let

$$
P_{\varepsilon}(D) G=\sum_{|\alpha| \leq m} a_{\alpha, \varepsilon} D^{\alpha} G=F_{\varepsilon}, \varepsilon \in(0,1)
$$

be a family of equations with constant coefficients and $F_{e} \in C^{\infty}(\Omega)$. If $P_{e}(D)$ is hypoelliptic for fixed $\varepsilon \in(0,1)$, then the corresponding solution to the above equation, $G_{\varepsilon}$, is in $C^{\infty}(\Omega)$. If we suppose that $\sup _{x \in K \subset \subset \Omega}\left|D^{\alpha} F_{e}(x)\right|$ satisfies the power growth condition $\mathcal{O}\left(\varepsilon^{-N_{K}}\right)$ for every $\alpha$ (for example, if $F_{\varepsilon}=f * \delta_{e}$, where $f \in \mathcal{D}(\Omega)$ and $\delta_{\varepsilon}$ is a delta net, and the above estimate holds, then $f \in C^{\infty}(\Omega)$ ), then the question which we want to answer is whether it is true that the derivatives of $G_{\varepsilon}$ satisfy similar estimates on compact sets.

The question is twofold important. First, the class $\left[F_{\varepsilon}\right]$ may represent a distribution or Colombeau's generalized function. Second, we investigate how the hypoellipticity depends on an appropriate perturbation of coefficients.

We implicitly answer these questions by putting the above family of equations into the space of Colombeau's generalized functions.

We recall necessary notions in order to present the main problems and results of the paper.

Let

$$
\begin{aligned}
& \mathcal{A}_{0}(\mathbb{R})=\left\{\phi \in C_{0}^{\infty} \mid \int \phi(x) d x=1, \operatorname{diam}(\operatorname{supp} \phi)=1\right\} \\
& \mathcal{A}_{q}(\mathbb{R})=\left\{\phi \in \mathcal{A}_{0} \mid \int x^{\alpha} \phi(x) d x=0,1 \leq \alpha \leq q, \alpha \in \mathbb{N}\right\}, q \in \mathbb{N}
\end{aligned}
$$


and

$$
\mathcal{A}_{q}=\mathcal{A}_{q}\left(\mathbb{R}^{n}\right)=\left\{\phi\left(x_{1}, \ldots, x_{n}\right)=\phi_{1}\left(x_{1}\right) \cdot \ldots \cdot \phi_{1}\left(x_{n}\right) \mid \phi_{1} \in \mathcal{A}_{q}(\mathbb{R})\right\}
$$

Put $\phi_{\mathrm{s}}=(1 / \varepsilon) \phi(\cdot / \varepsilon)$, where $\phi \in \mathcal{A}_{0}$.

Let $\Omega$ be an open subset of $\mathbb{R}^{n}$ and $\mathcal{E}(\Omega)$ be the space of functions $G: \mathcal{A}_{0} \times(0,1) \times \Omega \rightarrow \mathbb{C}$ which are $C^{\infty}$ for every $\phi \in \mathcal{A}_{0}$ and $\varepsilon \in(0,1)$. We will use the notation $G_{\phi, \varepsilon}$ for $(\phi, \varepsilon, x) \mapsto G_{\phi, \varepsilon}(x), x \in \Omega$.

It is said that a family of smooth complex valued functions on $\Omega, G_{\phi, e}, \phi \in \mathcal{A}_{0}$, $\varepsilon \in(0,1)$, belongs to $\mathcal{E}_{M}(\Omega)$ if for every compact set $K \subset \subset \Omega$ and $\alpha \in \mathbb{N}_{0}^{n}$ there exist $N \in \mathbb{N}$ and $r=r(K, \alpha) \in \mathbb{R}$ such that

$$
\sup _{x \in K}\left|\partial^{\alpha} G_{\phi, \varepsilon}(x)\right|=\mathcal{O}\left(\varepsilon^{\prime}\right), \varepsilon \rightarrow 0 \text {, for every } \phi \in \mathcal{A}_{N}
$$

If $G_{\phi, \varepsilon}$ does not depend on $x$ and (1) holds for $\alpha=0$, then the space of corresponding families of complex numbers is denoted by $\mathbb{C}_{M}$. For example, regularizing nets of distributions are elements of $\mathcal{E}_{M}$ : If $g \in \mathcal{D}^{\prime}$ the corresponding element in $\mathcal{E}_{M}$ is given by $G_{\phi, \varepsilon}=g * \delta_{\phi, \varepsilon}$, where we use the notation $\delta_{\phi, \varepsilon}=\phi_{\varepsilon}, \phi \in \mathcal{A}_{0}$ since it is a delta net.

The space of all elements $G_{\phi, \varepsilon}$ in $\mathcal{E}_{M}(\Omega)$ which satisfy (1) independently of $\alpha \in \mathbb{N}_{0}$ is denoted by $\mathcal{E}_{M}^{\infty}$.

Solutions to

$$
\sum_{|\alpha| \leq m} a_{\alpha, \phi, \varepsilon} D^{\alpha} G_{\phi, \varepsilon}=F_{\phi, \varepsilon}, F_{\phi, \varepsilon} \in \mathcal{E}_{M}, a_{\alpha, \phi, e} \in \mathbb{C}_{M}
$$

in $\mathcal{E}_{M}$ are constructed in [7], in a simplified version of Colombeau's theory, by adapting the classical distributional method of solving a constant coefficients partial differential equation. Problems which are specific for (2) are connected with the growth rate of solutions with respect to $\varepsilon$ which implies that the main assertions in [7] and in this paper are non-trivial generalizations of the corresponding ones in the space of distributions.

In this paper we investigate the hypoellipticity of the families of equations (2). This family is hypoelliptic if $F_{\phi, 8} \in \mathcal{E}_{M}^{\infty}$ implies $G_{\phi, 8} * \delta_{\phi, 8} \in \mathcal{E}_{M}^{\infty}$.

We note that the hypoellipticity of each equation of the family of equations (2) does not imply the hypoellipticity defined above, as it can be seen in the remark after Theorem 1.

The paper is organized as follows. First we recall the basic notions of Colombeau's generalized functions spaces (cf. [1, 5 and 7]). Then we give the necessary and sufficient conditions on $P(s)=\sum_{|\alpha| \leq m} a_{\alpha} s^{\alpha}$, where $a_{\alpha}$ are generalized constants, such that the corresponding operator $P(D)$ is hypoelliptic in $\mathcal{G}$ in Theorem 1 . We reformulate the necessary and sufficient conditions applying the theorem of Gorin ([3]) in Theorem 2. 


\section{Basic notions}

The space $\mathcal{E}_{0}(\Omega)$ (resp. $\mathbb{C}_{0}$ ) is defined to be the subspace of $\mathcal{E}_{M}(\Omega)$ (resp. $\mathbb{C}_{M}$ ) consisting of elements $G_{\phi, \varepsilon}$ such that for every $K \subset \subset \Omega, \alpha \in \mathbb{N}_{0}^{n}$ and $r \in \mathbb{R}$ there exists $N \in \mathbb{N}$ such that (1) holds (resp. (1) holds for $\alpha=0$ and $G_{\phi, 8}$ does not depend on $x$ ) for every $\phi \in \mathcal{A}_{N}$.

The space of Colombeau's generalized functions on an open set $\Omega \subset \mathbb{R}^{n}$ is defined by $\mathcal{G}(\Omega)=\mathcal{E}_{M}(\Omega) / \mathcal{E}_{0}(\Omega)$ and $\overline{\mathbb{C}}=\mathbb{C}_{M} / \mathbb{C}_{0}$ is the ring of Colombeau's generalized complex numbers. Note that $\Omega \rightarrow \mathcal{G}(\Omega), \Omega \subset \mathbb{R}^{n}$, is a sheaf.

$\left[G_{\phi, 8}\right]$ denotes the class in $\mathcal{G}$ (or $\overline{\mathbb{C}}$ ) determinated by the representative $G_{\phi, 8}$.

Let $G \in \mathcal{G}(\Omega)$. The complement of the largest open set of $\Omega$ in which $G$ is equal to the zero generalized function is called the support of $G, \operatorname{supp}_{g} G$.

The space of generalized functions with compact supports in the interior of $\Omega$ is denoted by $\mathcal{G}_{c}(\Omega)$.

$\mathcal{G}^{\infty}(\Omega)$ (cf. [5]) is the space of all generalized functions which have a representative in $\mathcal{E}_{M}^{\infty}$. It is a subalgebra of $\mathcal{G}(\Omega)$ and

$$
\mathcal{G}^{\infty}(\Omega) \cap \mathcal{D}^{\prime}(\Omega)=C^{\infty}(\Omega)(\text { see }[5])
$$

The space of tempered Colombeau's generalized functions $\mathcal{G}_{t}\left(\mathbb{R}^{n}\right)$ is defined to be $\mathcal{E}_{1}\left(\mathbb{R}^{n}\right) / \mathcal{E}_{0 t}\left(\mathbb{R}^{n}\right)$ where $\mathcal{E}_{1}\left(\mathbb{R}^{n}\right)$ is the set of all $G_{\phi, \varepsilon} \in \mathcal{E}$ such that for every $\alpha \in \mathbb{N}_{0}^{n}$ there exist $\gamma>0, N \in \mathbb{N}$ and $r \in \mathbb{R}$ such that

$$
\sup _{x \in \mathbb{R}^{n}}\left|\partial^{\alpha} G_{\phi, \varepsilon}(x)\right| /(1+|x|)^{y}=\mathcal{O}\left(\varepsilon^{r}\right), \varepsilon \rightarrow 0 \text { for every } \phi \in \mathcal{A}_{N}
$$

and $\mathcal{E}_{0 \mathrm{t}}\left(\mathbb{R}^{n}\right)$ is the space of all $G_{\phi, \varepsilon} \in \mathcal{E}_{\mathrm{t}}$ such that for every $\alpha \in \mathbb{N}_{0}^{n}$ there exists $\gamma \in \mathbb{R}$ such that for every $r \in \mathbb{R}$ there exist $N \in \mathbb{N}$ such that (3) holds for every $\phi \in \mathcal{A}_{N}$.

Note that $\mathcal{G}_{1}\left(\mathbb{R}^{n}\right)$ is not a subspace of $\mathcal{G}\left(\mathbb{R}^{n}\right)$, but there is a canonical map $\mathcal{G}_{1}\left(\mathbb{R}^{n}\right) \rightarrow \mathcal{G}\left(\mathbb{R}^{n}\right)$.

Let $G \in \mathcal{G}(\Omega)$. The complement of the largest open set of $\Omega$ in which $G$ is in $\mathcal{G}^{\infty}(\Omega)$ is called the singular support of $G$. It is denoted by singsupp, $G$.

The equality in $\mathcal{G}$ is often too strong for applications, so we shall use a notion of equality in generalized distribution (resp. generalized tempered distribution) sense $\stackrel{0 . d}{=}$ (resp. $\stackrel{g .1 .}{=}$ ) which is defined by:

$G_{1} \stackrel{g d .}{=} G_{2}$ (resp. $G_{1} \stackrel{g . t d .}{=} G_{2}$ for tempered generalized functions) if for every $\psi \in \mathcal{D}$ (resp. $z \psi \in \mathcal{S}),\left(G_{1}, \psi\right\rangle=\left\langle G_{2}, \psi\right\rangle$ in $\overline{\mathbb{C}}$, where $\langle G, \psi\rangle$ means $\int G(x) \psi(x) d x$.

Remark. The Dirac delta distribution $\delta$ is embedded in $\mathcal{G}$ as $\left[\delta_{\phi . e}\right]$ and for any $G \in \mathcal{G}, G *\left[\delta_{\phi, 8}\right]$ is represented by $G_{\phi, 8} * \delta_{\phi, \varepsilon}=\int G_{\phi, 8}(x-y) \delta_{\phi, 8}(y) d y$. There holds $G *\left[\delta_{\phi, l}\right] \stackrel{g . A .}{=} G$ and for every differential operator with constant coefficients $P(D)$, $P(D) G \stackrel{\theta d .}{=} P(D) G *\left[\delta_{\phi, \varepsilon}\right]$

By following [7], we define polynomials in $n$ real variables as elements of the ring 
$\overline{\mathbb{C}}\left[x_{1}, \ldots, x_{n}\right]$. A generalized polynomial function is a tempered generalized function of the form

$$
\sum_{|\alpha| \leq m} a_{\alpha} x^{\alpha}, a_{\alpha}=\left[a_{\alpha, \phi, e}\right] \in \overline{\mathbb{C}},|\alpha| \leq m
$$

It is of degree $m$ if $a_{\alpha}=0$ for $|\alpha|>m$ and there exists $\beta,|\beta|=m$ such that $a_{\beta} \neq 0$.

If $\left[H_{\phi, e}(x)\right]=\sum_{|\alpha| \leq m}\left[a_{\alpha, \phi, e}\right] x^{\alpha}$ is such a generalized function, then it can be written only in one way as a polynomial. In fact, if $\sum_{|\beta| \leq m} b_{\beta, \phi, e} x^{\beta}=N_{\phi, e}(x) \in \mathcal{E}_{0 t}\left(\mathbb{R}^{n}\right)$, then by making successive derivations and by putting $x=0$ it follows $b_{\beta, \phi, 8} \in \mathbb{C}_{0},|\beta| \leq m([7])$.

\section{Hypoelliptic differential operators}

Fundamental solution. Let us remember that in the classical distribution theory a fundamental solution of a differential operator is a distribution $E$ such that $P(D) E=\delta$.

Let

$$
P(D)=\sum_{|\alpha| \leq m} a_{\alpha} D^{\alpha}=\left[P_{\phi, \varepsilon}\left(i \frac{\partial}{\partial x}\right)\right]\left(D^{\alpha}=i^{|\alpha|} \partial^{\alpha}\right)
$$

where $\sum_{|\alpha| \leq m} a_{\alpha} x^{\alpha}$ is a polynomial in $\mathcal{G}$. In Colombeau's theory, the fundamental solution of $P$ is a generalized function $E \in \mathcal{G}$ satisfying $P(D) E=\left[\delta_{\phi, e}\right]$. This means that its representatives $E_{\phi, e} \in \mathcal{E}_{M}$ satisfy

$$
\sum a_{\alpha, \phi, 8} D^{\alpha} E_{\phi, e}(x)=\delta_{\phi, 8}(x)+N_{\phi, e}(x), x \in \mathbb{R}^{n},
$$

for some $N_{\phi, e} \in \mathcal{E}_{0}$.

This fundamental solution allows us to solve the equation $P(D) U \stackrel{\text { g.d. }}{=} G$ for $G \in \mathcal{G}$, because $G *\left[\delta_{\phi, \varepsilon}\right] \stackrel{g . d .}{=} G$.

Proposition 5. Let $P(D)$ be a generalized differential operator of the form (4) with coefficients in $\overline{\mathbb{C}}$ of degree $m$ such that for some $\left(c_{1}, c_{2}, \ldots, c_{n}\right) \in \mathbb{R}^{n}$ there exist $r>0$ and $N \in \mathbb{N}$ such that for every $\phi \in \mathcal{A}_{N}$ there exist $C>0$ and $\eta>0$ such that

$$
\left|\sum_{|\alpha| \leq m} a_{\alpha, \phi, \varepsilon} c^{\alpha}\right| \geq C \varepsilon^{r}, \varepsilon \in(0, \eta)
$$

Then, $P(D)$ admits a generalized fundamental solution.

In non-standard models of Colombeau's theory this hypothesis can be replaced by $\sum_{|\alpha|=m} a_{\alpha, \phi, c} c^{\alpha} \neq 0$, since $\overline{\mathbb{C}}$ is a field in such models (cf. $\mathrm{Li}$ Bang $\mathrm{He},[4]$ and Oberguggenberger [6]). 
Proof. Case 1 . Suppose that there exists $N \in \mathbb{N}$ such that for every $\phi \in \mathcal{A}_{N}$ there exists $\eta>0$ such that

$$
P_{\phi, \varepsilon}(D)=a_{m, \phi, \varepsilon} D_{1}^{m}+\sum_{k=0}^{m-1} P_{k, \phi, e}\left(D^{\prime}\right) D_{1}^{k}, \text { for } \varepsilon<\eta,
$$

where $D^{\prime}=\left(D_{2}, \ldots, D_{n}\right)$ and $D_{j}=i \frac{\partial}{\partial x_{j}}, j=1, \ldots, n$. There holds

$$
P_{\phi, \varepsilon}(s)=a_{m, \phi, e} s_{1}^{m}+\sum_{k=0}^{m-1} P_{k, \phi, e}\left(s^{\prime}\right) s_{1}^{k}=a_{m, \phi, e} \prod_{j=1}^{m}\left(s_{1}-s_{1, \phi, \varepsilon}^{j}\left(\sigma^{\prime}\right)\right),
$$

where $s^{\prime}=\sigma^{\prime} \in \mathbb{R}^{n-1}\left(\sigma^{\prime}=\left(\sigma_{2}, \ldots, \sigma_{n}\right)\right)$ is fixed, $s_{1}=\sigma_{1}+i \tau_{1} \in \mathbb{C}, s_{1, \phi, e}^{j}\left(\sigma^{\prime}\right)$ is the $j$-th root of $P_{\phi, \varepsilon}\left(s_{1}, \sigma^{\prime}\right)=0, \varepsilon \in(0, \eta)$ and $\phi \in \mathcal{A}_{N}$.

In this case (5) reads as follows: There exist $r \in \mathbb{R}$ and $N \in \mathbb{N}$ such that for every $\phi \in \mathcal{A}_{N}$ there exist $C_{1}>0$ and $\eta>0$ such that

$$
\left|a_{m, \phi, \varepsilon}\right| \geq C_{1} \varepsilon^{r}, \varepsilon \in(0, \eta), \phi \in \mathcal{A}_{N} .
$$

Let $\varepsilon \in(0, \eta)$ and $\phi \in \mathcal{A}_{N}$ be fixed as well as $\sigma^{\prime} \in \mathbb{R}^{n-1}$. Denote

$$
s_{1, \phi, \varepsilon}^{j}\left(\sigma^{\prime}\right)=\sigma_{1, \phi, \mathrm{e}}^{j}\left(\sigma^{\prime}\right)+i \tau_{1, \phi, \mathrm{e}}^{j}\left(\sigma^{\prime}\right), j=1, \ldots, m .
$$

There exists $k_{\sigma^{\prime}}=k_{\sigma^{\prime}, \phi, \varepsilon} \in\{0,1, \ldots, m+1\}$ such that $\left|k_{\sigma^{\prime}}-\tau_{1, \phi, \varepsilon}^{J}\left(\sigma^{\prime}\right)\right| \geq \frac{1}{2}$ for $j=1, \ldots, m$. This implies that

$$
\left|P_{\phi, \mathrm{s}}\left(\sigma_{1}+i k_{\sigma^{\prime}}, \sigma^{\prime}\right)\right| \geq \frac{C_{1} \varepsilon^{r}}{2^{m}}, \sigma_{1} \in \mathbb{R}
$$

where $C_{1}$ and $r$ are defined in (6) (and do not depend on $\varepsilon$ ). If $\tilde{\sigma}^{\prime} \in \mathbb{R}^{n-1}$, then

$$
P_{\phi, 8}\left(\sigma_{1}+i k_{\tilde{\sigma}^{\prime}}, \tilde{\sigma}^{\prime}\right)=a_{m, \phi, 8} \prod_{j=1}^{m}\left(\sigma_{1}+i k_{\tilde{\sigma}^{\prime}}-s_{1, \phi, 8}^{j}\left(\tilde{\sigma}^{\prime}\right)\right), s_{1} \in \mathbb{C} .
$$

Choose $\delta=\delta(m, \varepsilon)>0$ such that

$$
\left|s_{1, \phi, e}^{j}\left(\sigma^{\prime}\right)-s_{1, \phi, e}^{\prime}\left(\tilde{\sigma}^{\prime}\right)\right| \leq \frac{1}{2^{m+1}} \text { for }\left|\tilde{\sigma}^{\prime}-\sigma^{\prime}\right|<\delta .
$$

Then $\left|k_{\tilde{\sigma}^{\prime}}-\tau_{\phi .8}^{j}\left(\tilde{\sigma}^{\prime}\right)\right|>\frac{1}{2}-\frac{1}{2^{m+1}}$ and

$$
\left|P_{\phi, 8}\left(\sigma_{1}+i k_{\sigma^{\prime}}, \tilde{\sigma}^{\prime}\right)\right| \geq \frac{C_{1} \varepsilon^{\prime}}{4^{m}}
$$

Note that $\varepsilon$ and $\phi$ are fixed. We cover $\mathbb{R}^{n-1}$ by open cubes $L_{\sigma, \phi, e}, \sigma^{\prime} \in \mathbb{R}^{n-1}$. By the Heine-Borel theorem we can choose a sequence of open cubes $\Delta_{j, \phi, e}, j \in \mathbb{N}$, such that 


$$
\left|P_{\phi, e}\left(\sigma_{1}+i k_{j}, \sigma^{\prime}\right)\right| \geq \frac{C_{1} \varepsilon^{r}}{4^{m}}, \sigma_{1} \in \mathbb{R}, k_{j} \in\{0,1, \ldots, m+1\}, \sigma^{\prime} \in \Delta_{j, \phi, \varepsilon}
$$

Define

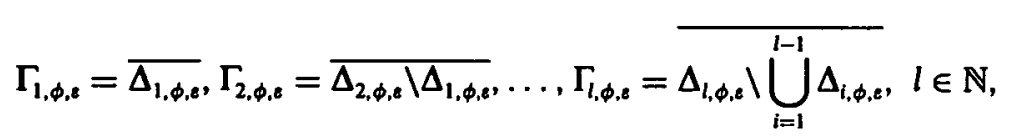

and $T_{\phi, 8}=\bigcup_{j=1}^{\infty}\left\{\left(\sigma_{1}+i \tau_{1}, \sigma^{\prime}\right) ; \sigma_{1} \in \mathbb{R}, \tau_{1}=k_{j} \in\{0, \ldots, m+1\}, \sigma^{\prime} \in \Gamma_{j, \phi, e}\right\}$.

Put

$$
E_{\phi, \mathrm{e}}(x)=\frac{1}{(2 \pi)^{n}} \int_{T_{\phi, 8}} \frac{e^{i\left(x,\left(\sigma_{1}+k_{1}, \sigma^{\prime}\right)\right)} \hat{\phi}\left(\varepsilon\left(\sigma_{1}+i \tau_{1}, \sigma^{\prime}\right)\right)}{P_{\phi, 8}\left(\left(\sigma_{1}+i \tau_{1}, \sigma^{\prime}\right)\right)} d s, x \in \mathbb{R}^{n}, \varepsilon \in(0, \eta)
$$

By a straightforward calculation one can prove that $E_{\phi, 8} \in \mathcal{E}_{M}$ and $P_{\phi, \varepsilon}(D) E_{\phi, s}=\delta_{\phi, \varepsilon}$.

Case II. Let $P_{\phi, 8}(x)$ be a representative of $P(x)$ such that its principal symbol $P_{m, \phi, e}(x)=\sum_{|x|=m} a_{\alpha, \phi, e} x^{\alpha}$ satisfies (5) for some $c=\left(c_{1}, \ldots, c_{n}\right) \in \mathbb{R}^{n}$, as it is assumed in the proposition.

Put $s_{j}=\sum c_{j k} t_{k}, s=C t$ such that $c_{1}=c_{11}, \ldots, c_{n}=c_{n 1}$ and other members are chosen so that the determinant of $C, \operatorname{det} C$, is not zero. Then,

$$
P_{\phi, 8}(s)=\tilde{P}_{\phi, 8}(t)=a_{m, \phi, t} t_{1}^{m}+\text { lower order terms, }
$$

where $\left|a_{m, \phi, \varepsilon}\right|=\left|P_{0 s}(c)\right| \geq C_{1} \varepsilon^{r}, \varepsilon \in(0,1)$ (cf. [6]).

Let $\tilde{E}_{\phi, 8}$ be a solution of $\tilde{P}_{\phi, \theta}(D) \tilde{E}_{\phi, 8}(x)=\frac{1}{g^{n}} \psi\left(\frac{x}{8}\right)$, where $\psi(x)=\phi\left({ }^{t} C^{-1} x\right)$ is constructed in the same way as in case I. Taking $\tilde{P}_{\phi, \mathrm{e}}(t)=P_{\phi, \mathrm{c}}(s), s=C t$, we obtain that the solution of $P_{\phi, \varepsilon}(D) E_{\phi, \varepsilon}=\frac{1}{e^{n}} \phi_{\varepsilon}$ is $E_{\phi, \varepsilon}(x)=\tilde{E}_{\phi, 8}\left({ }^{2} C x\right), x \in \mathbb{R}^{n}$.

Hypoelliptic differential operators. Let $\left[P_{\phi, 8}(D)\right]$ be of the form (4) and suppose that (5) holds for some $c=\left(c_{1}, \ldots, c_{n}\right) \in \mathbb{R}^{n}, C_{1}>0, r>0, N \in \mathbb{N}$ and $\eta>0$. This operator is called hypoelliptic if for every open $\Omega \subset \mathbb{R}^{n}$ and every solution $G \in \mathcal{G}(\Omega)$ to

$$
P(D) G=0,
$$

the generalized function $G *\left[\delta_{\phi, \varepsilon}\right]$ is in $\mathcal{G}^{\infty}(\Omega)$. In the introduction we gave this definition via the corresponding representatives.

As in the frame of distributions, we have the following two propositions.

Proposition 6. (a) $P(D)$ is hypoelliptic if it admits a fundamental solution $E$ with

$$
\text { singsupp } E=\{0\}
$$

(b) Let $\left[P_{\phi, 8}(D)\right]$ be hypoelliptic. Then, for every open set $\Omega$ and $G \in \mathcal{G}(\Omega), P(D) G \in$ $\mathcal{G}^{\infty}(\Omega)$ implies $G *\left[\delta_{\phi, \ell}\right] \in \mathcal{G}^{\infty}(\Omega)$. 
Proof. (a) Let $G \in \mathcal{G}(\Omega)$ be a solution of (7). Let $O$ be a neighbourhood of zero, $\alpha \in C_{0}^{\infty}(O)$ and $\alpha=1$ in some neighbourhood of zero contained in $O$. We shall prove that $G *\left[\delta_{\phi, \varepsilon}\right]$ is in $\mathcal{G}^{\infty}\left(\Omega_{0}\right)$, for every $\Omega_{0} \subset \subset \Omega$. Let $\beta \in C_{0}^{\infty}(\Omega), \beta=1$ on $\Omega_{0}$ and $W$ be an open subset of $\Omega_{0}$. We shrink $O$ such that $W-O \subset \Omega_{0}$. Then, for every $x \in W$, we have

$$
\begin{aligned}
G_{\phi, \varepsilon} * \phi_{\varepsilon}(x) & =P_{\phi, \varepsilon}(D)\left((1-\alpha) E_{\phi, 8}\right) *\left(\beta G_{\phi, \varepsilon}\right)+\left(\alpha E_{\phi, 8}\right) *\left(P_{\phi, \varepsilon}(D)\left(\beta G_{\phi, 8}\right)\right) \\
& =P_{\phi, e}(D)\left((1-\alpha) E_{\phi, \ell}\right) *\left(\beta G_{\phi, \varepsilon}\right)(x) .
\end{aligned}
$$

This implies $G *\left[\delta_{\phi, \ell}\right] \in \mathcal{G}^{\infty}(W)$ and thus $G *\left[\delta_{\phi, \ell}\right] \in \mathcal{G}^{\infty}\left(\Omega_{0}\right)$.

(b) It follows directly from (9).

Remark. Assertion (b) is usually used as the definition of hypoellipticity.

Let $P_{\phi, \varepsilon}$ be a hypoelliptic differential operator on $\Omega, \Omega_{0}, W$, and $O$ are the same as in the proof of assertion (a) in Proposition 6, $G$ a solution of (7) and $W \pm O \subset \Omega_{0}$. Then we have the following

Proposition 7. There exists $N \in \mathbb{N}$ such that for every $q \in \mathbf{N}_{0}^{n}$ there exist $C>0$ and $\eta>0$ such that

$$
\max _{x \in W}\left|D^{q} G_{\phi, \varepsilon} * \delta_{\phi, \varepsilon}(x)\right| \leq C \max _{x \in \Omega_{0}}\left|G_{\phi, \varepsilon}(x)\right| \varepsilon^{-N}, \varepsilon<\eta .
$$

Proof. Since this holds for $|q|=0$, assume $|q|>0$. Then, because of $\partial^{q}\left(P_{\phi, \ell}(D)\right.$ $\left.(1-\alpha(t)) E_{\phi, \varepsilon}(t)\right) \in C_{0}^{\infty}(O)$ for every fixed $\phi$ and $\varepsilon, \varepsilon$ small enough, we have

$$
\partial^{q} G_{\phi, e} * \delta_{\phi, \ell}(x)=\int_{0} \partial^{q}\left(P_{\phi, 8}(D)(1-\alpha(t)) E_{\phi, e}(t)\right) G_{\phi, e}(x-t) d t
$$

which implies

$$
\max _{x \in W}\left|\partial^{q} G_{\phi, \varepsilon} * \delta_{\phi, \varepsilon}(x)\right| \leq C \max _{x \in \Omega_{0}}\left|G_{\phi, \varepsilon}(x)\right| \max _{x \in O}\left|\partial^{q}\left(P_{\phi, e}(D)(1-\alpha(t)) E_{\phi, \varepsilon}(t)\right)\right|
$$

and thus the assertion follows.

Our main result is given in the theorem which is to follow and which is the extension of the corresponding well-known result in distribution theory. The idea of its proof is similar to the proof of the corresponding one for distributions given in $[2, p .304]$. The main difficulty comes from the fact that we need power order estimates concerning $V\left(P_{\phi, e}\right)$, where $V\left(P_{\phi, e}\right)=\left\{\sigma+i \tau \mid P_{\phi, \varepsilon}(\sigma+i \tau)=0\right\}$ is a set of zeros of $P_{\phi, s}$ for the fundamental solution $E_{\phi, e}$. Because of that, the use of [2, Lemma 3, pp. 303-304], crucial for the distributional proof, is not possible in our case. 
Theorem 1. The operator $P(D)$ is hypoelliptic if and only if there exist $N>0$ and $q \in \mathbb{N}$ such that for every $\phi \in \mathcal{A}_{q}$ and $A>0$ there exist $\eta>0$ and $B>0$ such that

$$
|\tau| \geq A(\log |\sigma|+N \log \varepsilon)-B, \sigma+i \tau \in V\left(P_{\phi, \varepsilon}\right), \varepsilon \in(0, \eta)
$$

Proof of necessity. Let $s \in \mathbb{C}^{n}$. Define a generalized function $G$ with a representative

$$
G_{\phi, s}=\left\{\begin{array}{l}
e^{-i(s, x)}, s \in V\left(P_{\phi, \varepsilon}\right) \\
0, \text { otherwise. }
\end{array}\right.
$$

Obviously $G \in \mathcal{G}^{\infty}$ and

$$
P_{\phi, \varepsilon}(D) G_{\phi, \varepsilon}=\left\{\begin{array}{l}
P_{\phi, \varepsilon}(D) e^{-i(s, x)}=P_{\phi, e}(s) e^{-i(s, x)}=0, s \in V\left(P_{\phi, \varepsilon}\right) \\
P_{\phi, \varepsilon}(D) 0=0, \text { otherwise, }
\end{array}\right.
$$

which means that $G_{\phi, 8}$ is a solution of (7). Since $e^{-i(s,)} \in C^{\infty}$, we have

$$
G_{\phi, 8}-\left(G_{\phi, 8} * \delta_{\phi, 8}\right)(x)=Z_{\phi, \varepsilon} \in \mathcal{N} \text {. }
$$

That means that for every compact set $K$ and $p \in \mathbb{R}$ there exist $N_{1} \in \mathbb{N}$ such that for every $\phi \in \mathcal{A}_{N_{1}}$ there exist $C_{1}>0$ and $\eta_{1}>0$ such that

$$
\sup _{x \in K}\left|Z_{\phi, \varepsilon}(x)\right| \leq C_{1} \varepsilon^{p}, \varepsilon<\eta \text {. }
$$

We apply Proposition 7 on $G_{\phi, 8}$ with $|q|=1, W=B(0, r)$ and $\Omega_{0}=B(0, R)$. If $s \in V\left(P_{\phi, s}\right)$, then we have

$$
\max _{x \in W}\left|D^{q}\left(e^{-i(s, y)} * \delta_{\phi, 8}(y)\right)(x)\right| \leq C \max _{x \in \Omega_{0}}\left|e^{-i(s, x)}\right| \varepsilon^{-N}, \varepsilon<\eta .
$$

This implies

$$
\max _{|x| \leq r}|s|\left|e^{-i(s, x)}+Z_{\phi, \varepsilon}(x)\right| \leq C e^{R|r|} \varepsilon^{-N}, \varepsilon<\eta
$$

and we have

$$
\max _{|x| \leq r}\left|s^{q} e^{-i(s, x)}\right| \leq C_{2} e^{R|\tau|} \varepsilon^{-N}
$$

for some $C_{2}>0$, because $Z_{\phi, \varepsilon} \in \mathcal{N}$ and for $K=B(0, R)$ one can suppose that $N=N_{1}$ and $\eta=\eta_{1}$ without loss of generality. Now

$$
|\sigma| \leq|s| \leq C_{2} e^{(R-r)|r|} \varepsilon^{-N}, \varepsilon<\eta
$$


which gives

$$
\log |\sigma| \leq \log C_{2}-N \log \varepsilon+(R-r)|\tau|
$$

i.e.

$$
|\tau| \geq\left(\log |\sigma|+N \log \varepsilon-\log C_{2}\right) /(R-r) .
$$

Since $R$ and $r$ can be arbitrarily chosen, the first part of the assertion follows.

Proof of sufficiency. We need to prove that (10) implies (8). The next lemma is needed for the estimation of integrals by which the fundamental solution is defined. It is analogous to [2, Lemma 3, pp. 303-304].

Lemma 1. Let $N, A, \eta$ and $B$ be the same as in (10). Assume that (a), (b) and (c) hold for $\varepsilon<\eta$, where

(a) $\left(\sigma_{1}, \sigma_{2}\right) \in \mathbb{R}^{2}$ such that

$$
A\left(\log \left|\left(\sigma_{1}, \sigma_{2}\right)\right|+N \log \varepsilon\right) \geq B+1 .
$$

(b) $\tau_{1} \in \mathbb{R},\left|\tau_{1}\right| \leq\left(A\left(\log \left|\left(\sigma_{1}, \sigma_{2}\right)\right|+N \log \varepsilon\right)-B\right) / 2$.

(c) $\left(\bar{\sigma}_{1}+i \bar{\tau}_{1}\right) \in V\left(P_{\phi, \ell}\right)$ and $\left|\bar{\tau}_{1}\right| \geq\left(A\left(\log \left|\left(\bar{\sigma}_{1}, \sigma_{2}\right)\right|+N \log \varepsilon\right)-B\right)$.

Then, for every fixed $\varepsilon<\eta$

$$
\left|\bar{\sigma}_{1}-\sigma_{1}\right| \geq \varepsilon^{N+1} \text { or }\left|\bar{\tau}_{1}-\tau_{1}\right| \geq \varepsilon^{N+1} .
$$

Proof. Fix $\varepsilon<\eta$. Suppose $\left|\bar{\sigma}_{1}-\sigma_{1}\right|<\varepsilon^{N+1}$. Then

$$
\begin{aligned}
\left|\bar{\tau}_{1}-\tau_{1}\right| & \geq\left|\bar{\tau}_{1}\right|-\left|\tau_{1}\right| \\
& \geq\left(A\left(\log \left|\left(\sigma_{1}, \sigma_{2}\right)\right|+N \log \varepsilon\right)-B\right) / 2-A\left(\log \frac{\left(\left|\sigma_{1}\right|^{2}+\left|\sigma_{2}\right|^{2}\right)^{1 / 2}}{\left(\left|\bar{\sigma}_{1}\right|^{2}+\left|\sigma_{2}\right|^{2}\right)^{1 / 2}}\right) .
\end{aligned}
$$

Since (a) implies $\left|\left(\sigma_{1}, \sigma_{2}\right)\right| \geq e^{(B+1) / A} \varepsilon^{-N}$, it follows that

$$
\left|\left(\bar{\sigma}_{1}, \sigma_{2}\right)\right| \geq e^{(B+1) / A} \varepsilon^{-N}-\varepsilon^{N+1} \geq e^{(B+1) / A} \varepsilon^{-N} / 2 .
$$

Put $r=A\left(\log \left(\frac{\left|\sigma_{1}\right|^{2}+\left|\sigma_{2}\right|^{2}}{\left|\bar{\sigma}_{1}\right|^{2}+\left|\sigma_{2}\right|^{2}}\right)^{1 / 2}\right) / 4$. Then (with $\left|\sigma_{1}\right|>\left|\bar{\sigma}_{1}\right|$ )

$$
\begin{aligned}
r & =A \log \left(1+\frac{\left(\left|\sigma_{1}\right|^{2}-\left|\bar{\sigma}_{1}\right|^{2}\right)}{\left(\left|\bar{\sigma}_{1}\right|^{2}+\left|\sigma_{2}\right|^{2}\right)}\right) / 8 \\
& =A \log \left(1+\left(\left|\sigma_{1}\right|-\left|\bar{\sigma}_{1}\right|\right) \frac{\left(\left|\sigma_{1}\right|+\left|\bar{\sigma}_{1}\right|\right)}{\left(\left|\bar{\sigma}_{1}\right|^{2}+\left|\sigma_{2}\right|^{2}\right)}\right) / 8 \\
& \leq(A / 8) \frac{\left(\left|\sigma_{1}\right|-\left|\bar{\sigma}_{1}\right|\right)}{\left|\left(\bar{\sigma}_{1}, \sigma_{2}\right)\right|} \\
& \leq A /\left(2 e^{(B+1) / A}\right)\left(\left|\sigma_{1}\right|-\left|\bar{\sigma}_{1}\right|\right) \varepsilon^{N} \leq A /\left(2 e^{(B+1) / A}\right) \varepsilon^{2 N+1}
\end{aligned}
$$


Thus, $\left|\bar{\tau}_{1}-\tau_{1}\right| \geq 1 / 2-\varepsilon^{2 N}$ for $\varepsilon<\eta$, which gives (11).

Proof of Theorem 1 (continuation). Now we will prove (8). We follow Friedman's proof [2, Theorem 4, p. 306] and add estimates with respect to $\varepsilon$. In fact we will give the exact estimates for $E_{j, \phi, s}$ constructed below for the part which corresponds to $j=2$. Other parts are briefly exposed.

We will assume, without loss of generality (as in the proof of Proposition 5), that $P_{\phi, 8}$ is of the form

$$
P_{\phi, \ell}(s)=a_{m, \phi, \varepsilon} s_{1}^{m}+\text { lower order terms. }
$$

We will give the proof in the case $n=2$. For $n>2$ the proof is the same but technically more complicated. The fundamental solution of $P_{\phi, e}$ is given by

$$
\begin{aligned}
E_{\phi, e}(x) & =(2 \pi)^{-n} \int_{T_{\phi, s}} \frac{e^{-i(x, s)} \hat{\phi}(\varepsilon s)}{P_{\phi, \varepsilon}(s)} d s \\
& =(2 \pi)^{-n} \sum_{k=1}^{\infty} \int_{\mathbf{R}} d \sigma_{1} \int_{\Gamma_{k, \phi, \varepsilon}} \frac{e^{-i\left(x,\left(\sigma_{1}+i \tau_{1}, \sigma^{\prime}\right)\right)} \hat{\phi}\left(\varepsilon\left(\sigma_{1}+i \tau_{1}, \sigma^{\prime}\right)\right)}{P_{\phi, 8}\left(\left(\sigma_{1}+i \tau_{1}, \sigma^{\prime}\right)\right)} d \sigma^{\prime},
\end{aligned}
$$

where $T_{\phi, s}=\bigcup_{j=1}^{\infty}\left\{\left(\sigma_{1}+i \tau_{1}, \sigma^{\prime}\right) ; \sigma_{1} \in \mathbb{R}, \tau_{1}=k_{j} \in\{0, \ldots, m+1\}, \sigma^{\prime}=\sigma_{2} \in \Gamma_{j, \phi, e}\right\}$ and $\Gamma_{j, \phi, e}$ are given in Proposition 5.

Let $\varepsilon<\eta$ be fixed. Divide the $\left(\sigma_{1}, \sigma_{2}\right)$ plane into nine regions $\Omega_{j}, j=1, \ldots, 9$ by the lines $\sigma_{1}= \pm \mu, \sigma_{2}= \pm \mu$ and denote them by $\Omega_{1}=\left\{\left|\sigma_{1}\right| \leq \mu,\left|\sigma_{2}\right| \leq \mu\right\}, \Omega_{2}=\left\{\sigma_{1} \geq \mu\right.$, $\left.\left|\sigma_{2}\right| \leq \mu\right\}, \Omega_{3}=\left\{\sigma_{1} \geq \mu, \sigma_{2} \geq \mu\right\}, \Omega_{4}=\left\{\left|\sigma_{1}\right| \leq \mu, \sigma_{2} \geq \mu\right\}, \ldots$ Choose $\mu>0$ such that

$$
A(\log \mu+N \log \varepsilon)-B \geq 1, A(\log \mu+N \log \varepsilon)-B \geq \max _{s \in T_{\phi . s}}|\tau| .
$$

Let $B_{r}=B\left(\left(x_{1}, x_{2}\right), r\right) \cap \mathbb{R}^{2} \backslash\{0\}$, where $r>0$. We will prove that $E_{\phi, s}$ represents an element in $\mathcal{G}^{\infty}\left(B_{r}\right)$. This implies the assertion of the theorem.

We will assume that $r$ is sufficiently small and that $B\left(\left(x_{1}, x_{2}\right), r\right) \subset[0, \infty) \times[0, \infty)$. Otherwise we first perform a suitable orthogonal transformation which does not restrict the proof.

Denote by $T_{j, \phi, 8}$ the projection of $\Omega_{j}$ on $T_{\phi, e}$. This means

$$
T_{j, \phi, \varepsilon}=\left\{\left(\sigma_{1}+i \tau_{1}, \sigma_{2}\right) \mid \sigma_{1} \in \mathbb{R}, \tau_{1} \in\{0, \ldots, m+1\}, \sigma_{2} \in \Gamma_{k, \phi, \varepsilon} \cap \Omega_{j}, k \in \mathbb{N}\right\} .
$$

Put

$$
E_{j, \phi, \varepsilon}(x)=(2 \pi)^{-n} \int_{T_{j, \phi, \varepsilon}} \frac{e^{-i\left(\left(x_{1}, x_{2}\right),\left(s_{1}, s_{2}\right)\right)} \hat{\phi}(\varepsilon s)}{P_{\phi, e}(s)} d s, j=1, \ldots, 9, x \in \mathbb{R}^{2}
$$

We will prove that all above generalized functions are in $\mathcal{G}^{\infty}\left(B_{r}\right)$. Clearly, $\left[E_{1, \phi, 8}\right] \in \mathcal{G}^{\infty}\left(B_{r}\right)$. 
Consider $\left[E_{2, \phi, 8}\right]$. The integration over the contour $\sigma_{1}+i \tau_{1}, \mu \leq \sigma_{1} \leq \nu, \tau_{1} \in\{0, \ldots, m+1\}$ is changed by integration over the contour

$$
\overline{Q(\mu) Q_{1}(\mu)} \cup \overline{Q_{1}(\mu) Q_{1}(v)} \cup-\overline{Q(v) Q_{1}(v)},
$$

where

$$
\begin{aligned}
& \overline{Q(\mu) Q_{1}(\mu)}=\left\{\mu+i t \mid 0 \leq t \leq \frac{1}{2}(A(\log \mu+N \log \varepsilon)-B)\right\}, \\
& \overline{Q_{1}(\mu) Q_{1}(v)}=\left\{\sigma_{1}+i \tau_{1} \mid \tau_{1}=\frac{1}{2}\left(A\left(\log \left|\sigma_{1}\right|+N \log \varepsilon\right)-B\right), \sigma_{1} \in[\mu, v]\right\}, \\
& \overline{Q(v) Q_{1}(v)}=\left\{v+i t \mid 0 \leq t \leq \frac{1}{2}(A(\log v+N \log \varepsilon)-B)\right\} .
\end{aligned}
$$

We have

$$
\begin{gathered}
E_{2, \phi, \varepsilon}(x)=(2 \pi)^{-n} \int_{-\mu}^{\mu}\left(\int_{\overline{Q(\mu) Q_{1}(\mu)}}+\int_{\overline{Q(v) Q_{1}(v)}}+\int_{\overline{Q_{1}(\mu) Q_{1}(v)}}\right) \\
\frac{e^{-i\left(\left(x_{1}, x_{2}\right),\left(s_{1}, s_{2}\right)\right)} \hat{\phi}(\varepsilon s)}{P_{\phi_{,}}(s)} d s=I_{1}+I_{2}+I_{3} .
\end{gathered}
$$

Since

$$
\left|P_{\phi, 8}\left(s_{1}, \sigma_{2}\right)\right|=\left|a_{m, \phi, 8}\right| \prod_{j=1}^{m}\left(\left|\sigma_{1}-\bar{\sigma}_{1}\right|^{2}+\left|\tau_{1}-\bar{\tau}_{1}\right|^{2}\right)^{1 / 2},
$$

where $\sigma_{2}$ is fixed, $\bar{\sigma}_{1}+i \bar{\tau}_{1} \in V\left(P_{\phi, 8}\right)$ and $s_{1}=\sigma_{1}+i \tau_{1}$ belongs to any of the quoted contours, Lemma 1 implies that $\left|P_{\phi, 8}(s)\right| \geq C \varepsilon^{r}$ on these contours for suitable $C>0$ and $r>0$.

One can easily prove that $\left[I_{1}\right] \in \mathcal{G}^{\infty}\left(B_{r}\right)$.

Since for every $k>0$ there exist $C_{k}>0$ and $C>0$ such that

$$
\left|\hat{\phi}\left(\varepsilon\left(v+i \tau, \sigma_{2}\right)\right)\right| \leq \frac{C_{k} e^{\ell|\tau|}}{\left(1+\varepsilon\left(v+\left|\sigma_{2}\right|^{2}\right)^{1 / 2}\right)^{k}} \leq C \varepsilon^{A N} v^{A / 2},
$$

it follows that $I_{3} \rightarrow 0$ as $v \rightarrow 0$.

So we have to prove that

$$
\left[(2 \pi)^{-n} \int_{-\mu}^{\mu} \int_{s_{1} \in I} \frac{e^{-i\left(x_{1}, s_{1}\right)} e^{-i\left(x_{2}, s_{2}\right)} \hat{\phi}\left(\varepsilon\left(s_{1}, \sigma_{2}\right)\right)}{P_{\phi, 8}\left(\left(s_{1}, \sigma_{2}\right)\right)} d s_{1} d s_{2}\right] \in \mathcal{G}^{\infty}\left(B_{r}\right),
$$

where

$$
I=\left\{s_{1}=\sigma_{1}+i \frac{1}{2}\left(A\left(\log \left|\sigma_{1}\right|+N \log \varepsilon\right)-B\right) \mid \sigma_{1} \in[\mu, \infty)\right\}
$$


Note, since $x_{1}>a$ for some $a>0$, there exists $C>0$ such that

$$
\left|e^{i\left(x_{1}, s_{1}\right)}\right| \leq C e^{-A x_{1} / 2\left(\log \left|\sigma_{1}\right|+N \log e\right)} \leq C \varepsilon^{-A a N / 2}\left|\sigma_{1}\right|^{-A a / 2} .
$$

Also, we have

$$
\left|d s_{1}\right| \leq\left(1+A /\left(a \sigma_{1}\right)\right) d \sigma_{1}
$$

and for every $k>0$ there exists $C_{k}>0$ such that

$$
\left|\hat{\phi}\left(\varepsilon\left(s_{1}, \sigma_{2}\right)\right)\right| \leq C_{k} \frac{e^{e\left(A\left(\log \left|\sigma_{1}\right|+N \log \varepsilon\right)-B\right) / 2}}{\left(1+\varepsilon\left(\left|\sigma_{1}\right|^{2}+\left|\sigma_{2}\right|^{2}\right)^{1 / 2}\right)^{k}}
$$

These inequalities simply imply that (12) holds.

Consider $E_{3 \varepsilon}$. It is determined by the integration over $\mu \leq \sigma_{1}<\infty$ and $\mu \leq \sigma_{2}<\infty$. By Cauchy's formula we change the integration over $T_{3, \phi, e}$ by integration over

$$
\left(\sigma_{1}+i\left(A\left(\log \left|\sigma_{1}\right|+N \log \varepsilon\right)-B\right) / 2\right) \times \sigma_{2}, \sigma_{1} \in[\mu, \infty), \sigma_{2} \in[\mu, \infty),
$$

and then by integration over

$$
\begin{gathered}
\left(\sigma_{1}+i\left(A\left(\log \left|\sigma_{1}\right|+N \log \varepsilon\right)-B\right) / 2\right) \times\left(\sigma_{2}+i\left(A\left(\log \left|\sigma_{2}\right|+N \log \varepsilon\right)-B\right) / 2\right), \\
\sigma_{1} \in[\mu, \infty), \sigma_{2} \in[\mu, \infty) .
\end{gathered}
$$

By (11) and the suitable estimates for $\hat{\phi}$ and $i\langle x, s\rangle$, one can prove that $\left[E_{3 \varepsilon}\right] \in \mathcal{G}^{\infty}\left(B_{r}\right)$. The proof that $\left[E_{k, \phi, e}\right] \in \mathcal{G}^{\infty}\left(B_{r}\right), k=4, \ldots, 9$ is the same as for $k=2$ or $k=3$.

Remarks. 1. The perturbation of the non-hypoelliptic operator $\partial_{x}$ leads to a family of the operators $\partial_{x}+\varepsilon \partial_{y}^{2}$. One can check that this family does not satisfy the assumption (10) in Theorem 1. So, this family of differential operators is not hypoelliptic, although $\partial_{x}+\varepsilon \partial_{y}^{2}$ is hypoelliptic for every fixed $\varepsilon$.

2. The operator $\partial_{x}^{2}$ is not hypoelliptic while the family $\partial_{x}^{2}+\varepsilon \partial_{y}^{2}$ is hypoelliptic in the above sense.

A usual relation describing the hypoellipticity of a differential operator is given in the next theorem.

Theorem 2. An operator $\left[P_{\phi, e}(D)\right]$ is hypoelliptic iff there exist $N>0$ and $q \in \mathbb{N}$ such that for every $\phi \in \mathcal{A}_{q}$ there exist $h>0, \eta>0$ and $b \in \mathbb{R}$ such that

$$
\sigma+i \tau \in V\left(P_{\phi, \varepsilon}\right) \Rightarrow|\tau| \geq \varepsilon^{N h}|\sigma|^{h}-b, \varepsilon \in(0, \eta) .
$$

Proof. Clearly, (13) implies (10). Assume that (10) holds, that $\varepsilon<\eta$ and $\phi \in \mathcal{A}_{q}$ are fixed. We will use the following theorem of Gorin [3, Theorem 4]. 
Let $P\left(\sigma_{1}, \sigma_{2}, \sigma_{3}\right)$ be a real polynomial of $n=n_{1}+n_{2}+n_{3}$ variables $\sigma_{i} \in \mathbb{R}^{n_{i}}, n_{i} \in \mathbb{N}_{0}$, $i=1,2,3$. If the surface $\mathcal{M}$, given by $P\left(\sigma_{1}, \sigma_{2}, \sigma_{3}\right)=0$, is not empty and lies in the domain $\left|\sigma_{2}\right| \geq \varphi\left(\left|\sigma_{1}\right|\right)$, where $\varphi(t) \rightarrow \infty$ as $t \rightarrow \infty$, then there exist $h>0$ and $b>0$ such that $\mathcal{M}$ lies in the domain defined by

$$
\left|\sigma_{2}\right| \geq\left|\sigma_{1}\right|^{h}-b .
$$

First, we note that

$$
\sigma+i \tau \in V\left(P_{\phi, \ell}\right) \Leftrightarrow(\sigma, \tau) \in \tilde{V}\left(P_{\phi, e}\right),
$$

where $\tilde{V}\left(P_{\phi, c}\right)$ is the set of real zeros of

$$
\tilde{P}_{\phi, \ell}(\sigma, \tau)=P_{1, \phi, 8}^{2}(\sigma, \tau)+P_{2, \phi, \ell}^{2}(\sigma, \tau) \text { and } P_{\phi, \ell}(\sigma+i \tau)=P_{1, \phi, 8}(\sigma, \tau)+i P_{2, \phi, e}(\sigma, \tau) \text {. }
$$

The polynomial

$$
\varepsilon^{M} P_{\phi, \varepsilon}(\sigma+i \tau)=\varepsilon^{M} P_{1, \phi, \varepsilon}(\sigma, \tau)+\varepsilon^{M} i P_{2, \phi, \varepsilon}(\sigma, \tau)
$$

has the same set of zeros as $P_{\phi, \varepsilon}$ for every $\varepsilon \in(0, \eta), \eta$ small enough.

Put $\tilde{\tilde{P}}_{\phi, \varepsilon}(\sigma, \tau)=\tilde{P}_{\phi, \varepsilon}\left(\varepsilon^{N} \sigma, \tau\right)$. Then (10) implies

$$
(\sigma, \tau) \in \tilde{\tilde{V}}\left(P_{\phi, \varepsilon}\right) \Rightarrow\left(\varepsilon^{-N} \sigma, \tau\right) \in \tilde{V}\left(P_{\phi, \varepsilon}\right) \Rightarrow|\tau|>A \log |\sigma|-B .
$$

Thus Gorin's theorem implies that there exist $h$ and $b$ such that $|\tau| \geq|\sigma|^{h}-b$ for $(\sigma, \tau) \in \tilde{V}\left(P_{\phi .8}\right)$. This implies

$$
|\tau| \geq \varepsilon^{N h}|\sigma|^{h}-b \text { if }(\sigma, \tau) \in \tilde{V}\left(P_{\phi, \delta}\right),
$$

which is desired assertion.

Acknowledgement.The authors would like to express their gratitude to $\mathbf{M}$. Oberguggenberger for his valuable remarks concerning the paper.

\section{REFERENCES}

1. J. F. Colombeau, Elementary Introduction in New Generalized Functions (North Holland, Amsterdam, 1985).

2. A. Friedman, Generalized unctions and Partial Differential Equations (Prentice-Hall, INC, Englewood Cliffs, New York, 1963).

3. E. A. Gorin, Asymptotic properties of polynomials and algebraic functions of several variables, Russian Math. Surveys (1961), 93-119.

4. Li Bang-He, Non-standard analysis and multiplication of distributions, Sci. Sinica 21 (1978), 561-585. 
5. M. Oberguggengerger, Multiplication of Distributions and Applications to Partial Differential Equations (Pitman Res. Not. Math. 259, Longman Sci. Techn., Essex, 1992).

6. M. Oberguggenberger, Products of Distributions: Nonstandard Methods, Z. Anal. Anwendungen 7 (12) (1988), 347-365.

7. S. Pilipović and D. SCARPalÉzos, Differential operators with generalized constant coefficients, Portugal. Math. 53 (3) (1996), 305-324.

UNIVERSITY OF NOVI SAD

FACULTY OF SCIENCE

INSTITUTE FOR MATHEMATICS

TRG D. OBRADOVIĆA 4

Novi SAD

Yugoslavia 